\title{
Personal Iris Recognition from an Image at Long Distance using Back Propagation Neural Network
}

\author{
Swati D. Shirke, C.Rajabhushanam
}

\begin{abstract}
Now days, most reliable system for person identification is Iris recognition technique. Most of other systems are also presents for person identification like as identification cards or tokens, secret codes, passwords, etc. But the problems of these types of systems are, the secret codes and passwords can be cracked, the identification cards can be damaged. Therefore the effective method for the person identification is necessary. The iris recognition is treated as the most accurate method for personal identification. The person's biometric physical and behavioral features are considered for the identification. This is most efficient technique, because the iris characteristics of a person cannot be change due to the age and environment. Therefore automatic systems are based on the iris recognition. In this paper, an effective technique for iris recognition is present to identify the individual. For the implementation of this system, the iris images are taken from UBIRIS.v1 database. This iris image is then segmented, normalized and features are extracted by using Hough Transform, Daugman rubber sheet modal and median filter. The matching of an iris is done with the help of back propagation neural network model. Also, the Chronological Monarch Butterfly Optimization -based Deep Belief Network (Chronological MBO-based DBN) is proposed for iris recognition to get better accuracy. All these operations are done on the MatLab.
\end{abstract}

Keywords : Back Propagation Neural Network, Cronologival Neural Network, Deep Belief Network(DBN), Iris Recognition, Image Segmentation, Biometric Identification.

\section{INTRODUCTION}

Now a days, in world most of the countries faces the problem of fake identity. This is a very serious problem and it is quite difficult to identify the fake person. But the biometric system can solve the above problems. Therefore most of the countries uses biometric system for security purpose such that in custom clearance, airport boarding, congregation entrance and so on. The Indian government also uses biometric system for identification of citizen in different applications like as in rashan shop, Aadhar project, in different government exam forms and registration dept. etc. There are different types of biometrics are presents such as voice, palm, finger, face, DNA, etc. But the iris recognition is the most accurate and stable biometric system for individual identification. Because the Iris is a unique thing of a person, it does not change with time and environment. It remains fixed and constant.

Revised Manuscript Received on July 10, 2019.

Ms. Swati D. Shirke, Ph.D.Scholor, Bharath Institute of Higher Education and Research Chennai, Tamil Nadu, India .Email:shirke.swati14@gmail.com

Dr. C.Rajabhushanam, Professor, Computer Science \& Engineering, Bharath Institute of Higher Education and Research, Chennai, Tamil Nadu, India., rajabhushanamc.cse@bharathuniv.ac.in throughout the life of person. Also the error rate, applicability and accuracy of iris recognition system better than the other biometric systems. Therefore for achieving and maintaining security, the iris recognition system plays an important role.

The portion lies in between the white sclera and the pupil of eye is called iris texture or iris region. Different features of an eye are presents in this iris region. These features are crypts, furrows, stripes, coronas, freckles, etc. These features are vary to person to person. The irises of identical twins are also entirely dissimilar. Figure 1 shows the different features of an eye.In a literature survey different encoding techniques are presents, but in case of matching of an iris image stage very less work is present. Therefore in this paper a successful matching is done with the help of deep neural network. The deep neural network is a pattern recognition method which classifies the data. The main goal is to design a system which is capable to classify different iris images. The superb-resolution technique is used to get the information from iris image. The superb-resolution technique is the most important technique and it can get the information from poor quality iris image. To solve the iris recognition and image processing problems, the localization of pupil and iris region a Hough transform is used with the help of normalization and automatic segmentation. And for the normalization and automatic segmentation purpose a Daugman's Rubber Sheet Model is used. The iris features are effectively extracted by using the ScatT-Loop descriptor and the Local Gradient Pattern (LGP), which is fed to the Chronological MBO-based $\mathrm{DBN}$ for iris recognition that enhances the accuracy.

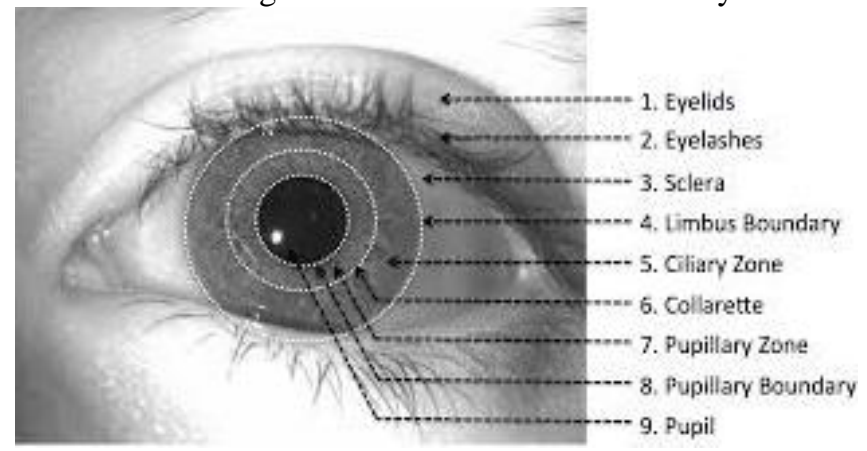

1. Features of eye [1].

The iris recognition and image processing problems can be solved by using Hough transform the localization of pupil and iris region is achieved with the help of normalization and automatic segmentation. For iris image normalization the 
Daugman's Rubber Sheet Model is designed.

Following is the structure of the current work. The related work is presents in the section 2 . In section 3 , the flow of proposed system is present. The building blocks of proposes structure is presents in section 4. The experimental results and the discussion are offered in Section 5. Conclusions are provided in Section 6.

\section{RELATED WORKS}

In last decade, most of the researchers are work on the iris recognition systems for the identification of person. They use different methods for localizing the iris. An effective algorithm designed by Daugman [2] for iris recognition. The internal and external borders iris image is detected via Integro-differential operators by using this Daugman algorithm. The 2DGabor wavelets filters are used for the feature extraction and for demodulation of iris's texture construction. It shows 1024 complex values of an iris image after applying the set of filters. Then each of these values quantized into complex plane of four quarters. And then the Hamming distance is calculated.

The weighted gradients are used in the paper Masek and Kovesi [3] for the iris recognition. The circular Hough-transform and modified canny edge detector are also used in this paper.

The iris conformity depending on two variation functions and the Hough transform to localization the iris are used by Boles [4].

The pyramid of Laplacian with four resolution levels are used to produce the code of the iris and Hough transform is used to localize the iris is employed in Wildes [5].

The different features of an iris image are extracted in the paper written by Ma, Wang, and Tan [6]. They use 2D Haar wavelet used to perform the matching process.

In a paper [7], A. E. Hassanien, A. Abraham, and C. Grosan identified patterns of iris via ICA coefficients, the reasonable learning device for identification of center of each class and the Euclidean distances are used to recognize the pattern of iris. Also in this paper the different noises like as noises occurs due to light are removed from eyelashes and eyelids. The blurred iris image is also accurately recognized by this method.

The globular Hough transform are presented in [8] by O. F. Soylemez, and B. Ergen are used for recognition purpose.

The internal and external borders of the iris image area were extracted in paper Altunkaya and Abiyev [9]. The normalization and enhancement algorithms are also presents in this paper to extract the different features of an eye image and embodied. To classify the iris patterns of iris image, an ANN classifier is designed in this paper. An adaptive learning tactic

is used to train the ANN classifier. The experimental results obtained from this ANN classifier shows the proficient identification of individuals.

An iris identification technique that is based on distinct wavelet covariance employing competitive ANN presented in Leila, et al, [10]. The construct a covariance matrix by means of distinct wavelet transform by ANN are used to identify a group of boundaries of iris profiles.

A cascade forward back propagation ANN model (CFBPNN) and an FFBPNN model were used to identify patterns of the iris image in paper written by Gopikrishnan and Santhanam [11]. Based on their results, they concluded that the CFBPNN model is more proficient than the FFBPNN model.

On the other hand, K. A. Raghavi, M. V. Priya, G. P. M. Paiva et al., [12] formed an better system of iris recognition with the purpose be able to overcomes the limits of individual's identification approaches. They employed a speedy algorithm for the reason of locating the area of the iris. They employed an indefinite neural network algorithm to obtain iris deterministic shapes taking the form of feature routes. These characteristic features can be equate based on weighted hamming distance to show the individuality. They adopted a twofold coding system to achieve more efficiency.

\section{Flow OF Proposed SySTEM}

\section{A. Image Acquisition}

Iris imaging at a distance for an optical system design is critical. Therefore for iris image acquisition, the lens based on the geometry optics and the required parameters of camera are calculated. Let the height of person is $\mathrm{H}$, the digital sensor's width as $\mathrm{W}$ and focus of lens are indicated by F, the object-image ratio as $\mathrm{M}$ and sensor pixel size as $\mathrm{S}$.
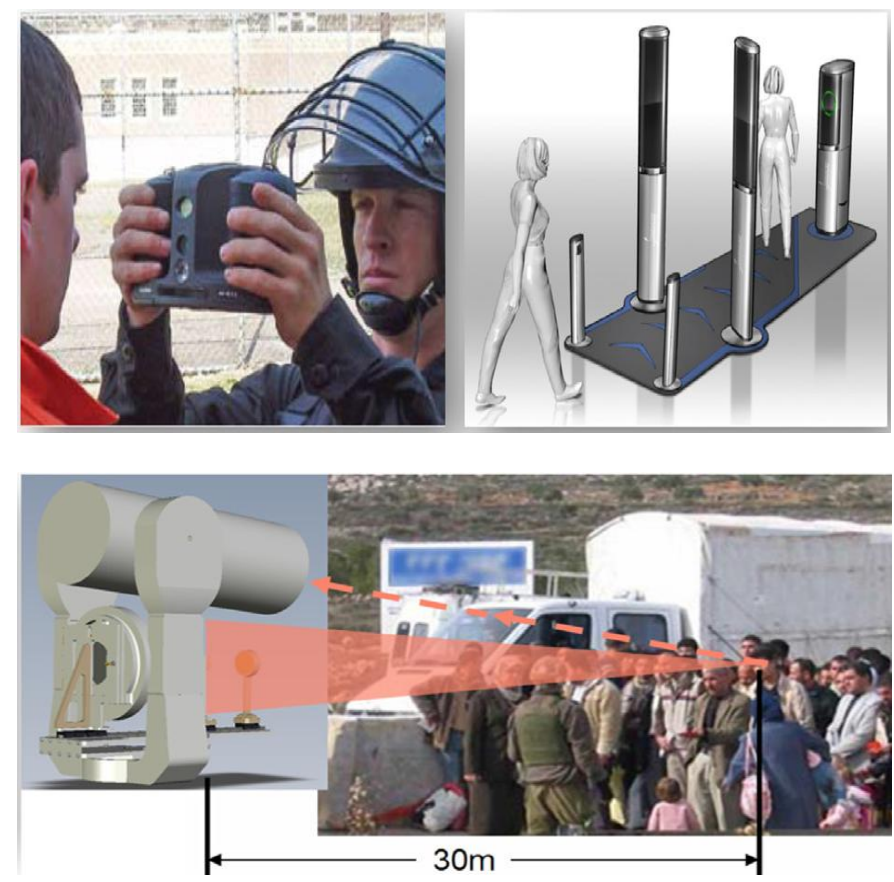

Fig.2 Different iris recognition systems examples close-range and long-range.

The distance D of an objected can be calculated from equation 1.

$$
D=F \frac{M}{S}
$$

The equation 2 shows the field depth in which the capturing volume is $\mathrm{V} \times \mathrm{A}$. 


$$
\begin{aligned}
& V=M W \\
& A=M H \\
& B=2 F \frac{(M-S)(M-S)}{S}
\end{aligned}
$$

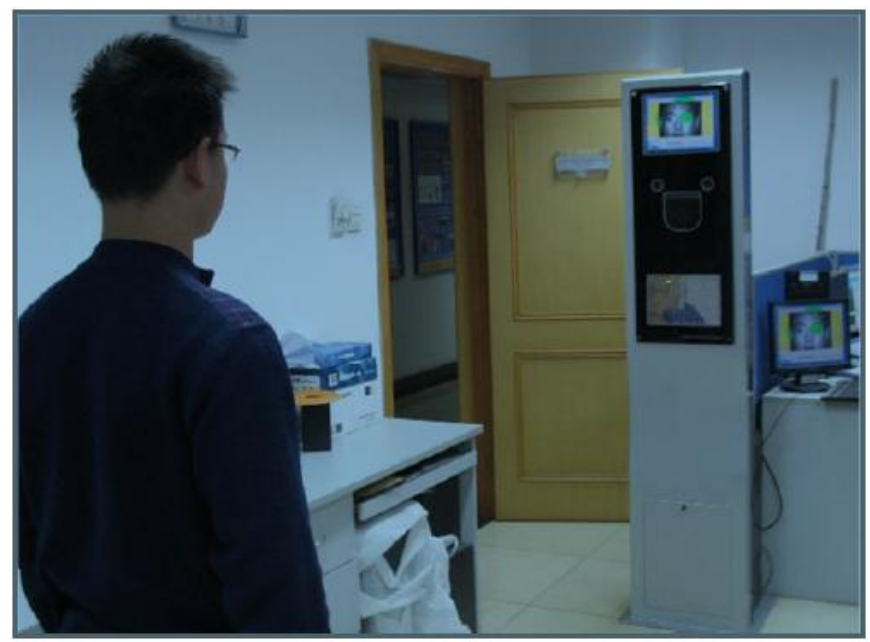

Fig.3 Process Of Capturing The Human Face Image.

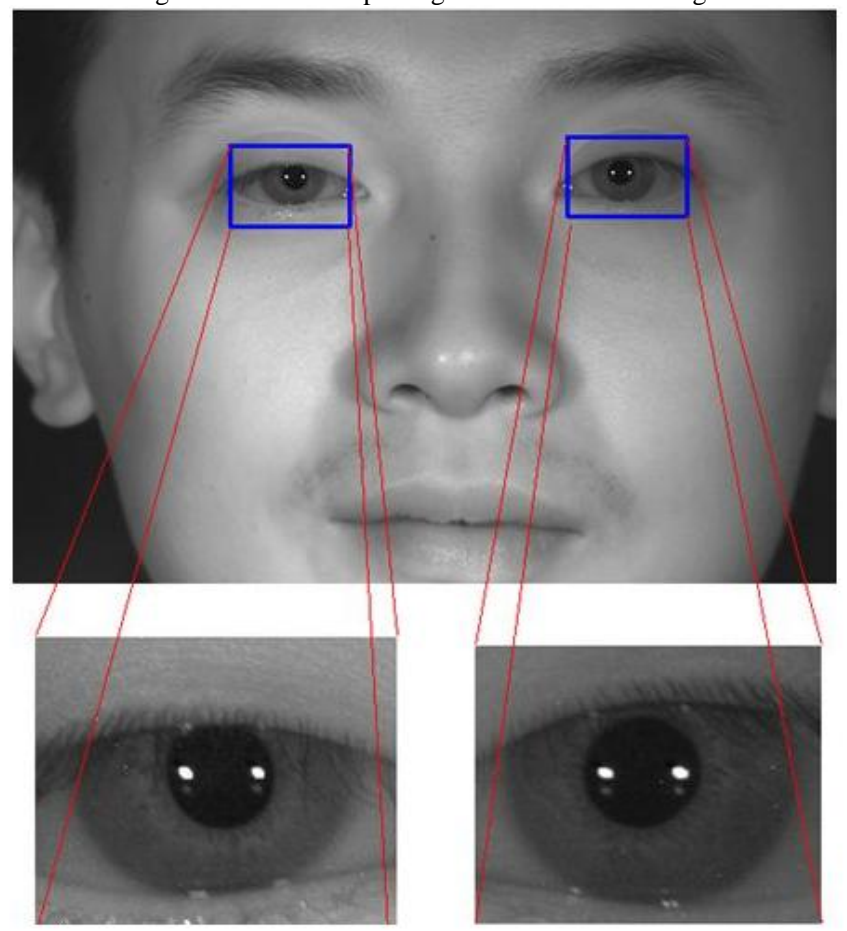

Fig.4 Process Of Extracting The Eye From The Face.

By considering the above geometry optics, the lens and the camera used in this article with frame rate of 30 frames per second and the pixel size is 4-mega pixels. This camera can takes a picture of human face completely with high-resolution. The lenses with its aperture size $\mathrm{F}$ is 15 and focal length $300 \mathrm{~mm}$. So it can capture the iris image clearly up to distance $4 \mathrm{~m}$ to $6 \mathrm{~m}$. Figure 2 shows different iris recognition systems examples close-range and long-range.

UBIRIS.v1 are the iris images which are captured from a distance from 3 meters away by actively searching iris, face or palmprint patterns. Therefore in this article, the UBIRIS.V1 database are used for the iris recognition. The process of capturing the human face image is shown in figure 3. In figure 4 the process of extracting the eye from the face is presents.

\section{B. Processing of an Image}

The quality of iris image obtained from the camera is poor. This can be happen due to the motion of person, distance, blur or occlusions. Therefore it is necessary to pre-process the iris image to enhance the different parameters an iris image such as contrast, intensity, signal to noise ratio, etc. for further processing. The technique which is used to enhance the iris image parameters are contrast stretching and histogram equalization. It only enhances the quality of image, it does not increases the information content of an image. The intensity range of an iris image is normalized to [0 1]. The signal to noise ratio of iris image is improved with the help of anisotropic diffusion filter. The tool which is used for this purpose is MatLab realize the many brightness transformations. To find the rank-order information and spatial information of an iris image a weighted median (WM) filter is used and this is one of the type of median filter. The noises shot and impulse noise are rejected by the median filter.

\section{Segmentation}

In a segmentation step the inner and outer boundaries of the iris region are detected. This can achieved by Hough transform. The Hough transform finds different shapes like as circles or ellipses of an image. It is the first step in the Iris recognition system also the backbone of the complete recognition system. It aims to detect layout, centers, eyelids, eyelashes and radii, of the two iris borderline. Locating the lower as well as upper eyelid also separate eyelashes. With the help of the Daugman's Rubber Sheet Model the image segmentation, acquisition and feature encoding of an iris image can takes place. It is also used to improve the quality of edges presents in the image. The most commonly used circle detector is integro-differential operator and it is mathematically expressed as.

$M A X / G(r) * \frac{d}{d r} \int \frac{I(x, y)}{2 \pi r}$

Where I ( $\mathrm{x}, \mathrm{y}$ ) denotes the input iris image, $\mathrm{G}(\mathrm{r})$ denotes Gaussian with a standard deviation, $r$ is the radius of circular arc. It is the convolution operation which is shown in symbol *. The line an iris image can be detected by the formula

$$
r=x \cos \theta+y \sin \theta
$$

where, $r$ is quantized distance and $\theta$ is quantized angle.

The $r$ and $\theta$ are considering quantized values in the pair $(r, \theta)$.

The boundary of the inner pupil and outer pupil of an iris image can be detected by using the equation,

$$
\left(m-m_{0}\right)^{2}+\left(p-p_{0}\right)^{2}=a^{2}
$$

where, $\left(m_{0}, p_{0}\right)$ denotes the coordinates of a circle with radius a. The results obtained from Hough transform shows the boundary of pupil, eyelid extraction of iris image and the center of pupil.

\section{Normalization}

In this the segmented iris image is normalized into the block with equal in size respect to the block width $x$ and angular displacement $\theta$. The 
Daugman's rubber sheet is an linear model that is assigned to the iris of the individual pixel based on the dilation, size and the real coordinates $(\mathrm{x}, \theta)$, where $\mathrm{x}$ is the unit interval and $\theta$ ranges from 0 and $2 \pi$. The iris image is remapped into the polar coordinate $(\mathrm{x}, \theta)$ system from cartesian coordinated $(\mathrm{r}$, s) system. Therefore the normalized polar coordinates are (X, $\theta)$ and the normal coordinates are (r, s).

Let the iris coordinates and iris boundaries of the pupil are represented as $\left(\mathrm{r}_{\mathrm{b}}, \mathrm{s}_{\mathrm{b}}\right)$ and $\left(\mathrm{r}_{\mathrm{e}}, \mathrm{s}_{\mathrm{e}}\right)$ along $\theta$ direction. The coefficient of an iris ime age will not be shifted even if the signal is distorted due to the camera and persons position.

\section{Feature Extraction \& Feature Matching using ScatT-loop}

In feature extraction step, the iris image is classified into new vessels image. The normalized iris image is subjected to perform the feature extraction by using ScatT-loop descriptor. The ScatT-loop generates the texture features for accurate iris recognition to uniquely identify the individuals. In this, the image region contains many vessel segments that are closely spaced with multiple orientations and have a twisted in nature. So for the measurement of feature characteristics, the new vessel segments are generated from a binary vessel maps. In order to find out local features, a sub window of size $4 \times 4$ is created. The iris image are examined through this sub window. And for every sub window the number of vessel pixels and pixel passion can calculated. The Kirsch mask is designed for the future extraction. The loop value for the corresponding pixel is represented as,

$$
\operatorname{LOOP}\left(r^{*}, s^{*}\right)=\sum_{k=0}^{7} h\left(G^{k}-G^{*}\right) \cdot 2^{v}
$$

Where,

$$
h(d)=\left\{\begin{array}{lll}
1 & ; & \text { if } d \geq 0 \\
0 & ; & \text { Otherwise }
\end{array}\right.
$$

$\left(r^{*}, s^{*}\right)$ is the centre of the intensity of iris image. $\mathrm{G}_{\mathrm{k}}$ is the neighborhood pixel intensity, $\mathrm{G}$ is the original image pixel intensity, and $k$ takes the value ranges from 0 to 7 .

\section{Extraction of features using Local Gradient Pattern (LGP)}

The LGP generates the constant patterns of face representation of person, which is irrespective to the intensity variation with the edges. The intensity values of a pixel are determined by gradient pixel values which is operated by LGP operator. The minimum value of the gradient among the eight neighboring pixels is considered as the threshold value. When the gradient value of the neighboring pixel is higher against the threshold, then the value assigned to the pixel is ' 1 ' otherwise the value is ' 0 '. The LGP can be represented as the formula

$$
L G P_{b, m}\left(r_{a}, s_{a}\right)=\sum_{i=0}^{b-1} p\left(y_{i}-\bar{y}\right) 2^{n}
$$

where,

$p(r)= \begin{cases}0, & \text { if } r<0, \\ 1, & \text { otherwise. }\end{cases}$ $m$ is the centre of a circle, $b$ are the sample points on the circle, LGP uses the bilinear interpolation to compute the neighborhood pixel values for $m$ and $b$. It uses $(2 \times m+1)$ and $(2 \times m+1)$ kernel to summarize the structure of the iris. Gradient value between the neighboring pixel $n_{i}$ and the center pixel $n_{a}$ is denoted as, $y_{c}=\left|n_{i}-n_{a}\right|$ and define the average gradient value of $b$ as,

$$
\bar{y}=\frac{1}{b} \sum_{i=0}^{b-1} y_{i}
$$

The feature vector is represented as,

$$
Y=\left\{Y_{1}, Y_{2}, \ldots . Y_{w}\right\} ; \text { for }(1<\tau<w)
$$

where, dimension of features computed from the input image $X_{i}$ is represented as [ $1 \mathrm{x} \mathrm{w}$ ] and $\mathrm{w}$ is the total feature dimension.

\section{Principal Component Analysis (PCA)}

Principal Component Analysis (PCA) is a method which is used to decrease the dimensionality of an image. This method is also used for multivariate analysis of an image. PCA is a compression technique which compresses the high dimensional vectors of an image into the low dimensional vectors and compute the parameters from the data directly. To reduce the dimensionality of an image, PCA extracts less number of component. The principal components of an image can be extracts by using covariance matrix or multivariate set. The compression and decompression operations of an image ca perform by using matrix multiplication. The PCA model is represented as,

$$
z_{o c 1}=D_{o c w} c_{w c 1}
$$

Where, $\mathrm{z}$ is an zero dimensional vector with the projection $\mathrm{c}$, and $\mathrm{w}$ is the feature vector dimension as $(0<\mathrm{w})$. The covariance matrix $\mathrm{E}$ is denoted as,

$$
E=\frac{1}{\rho-1} \sum_{\tau=1}^{\rho}(c-\kappa)(c-\kappa)^{G}
$$

where, $K$ denotes the mean vector of $C$. The Eigen vectors $\beta_{\mathrm{T}}$ is expressed as,

$$
\left(E-\phi_{\tau} \vartheta\right) \beta_{\tau}=0 \quad ; \quad \tau=1,2, \ldots \ldots . w
$$

Where, $\varnothing_{\mathrm{T}}$ denotes the Eigen vectors of $E$. The projection matrix is calculated as,

$$
D=J^{G}
$$

where, $J$ has 0 Eigen vectors and $\mathrm{D}$ is the ocw matrix. The dimensionally reduced feature vector is represented as, $\gamma=\left\{\gamma_{1}, \gamma_{2}, \ldots ., \gamma_{\tau} \ldots . \gamma_{o}\right\} ;$ for $(1<\tau<o)$

where, 0 is the dimensionally reduced features with $0<w$.

\section{BPNN Based Iris Recognition}

For categorization purpose the artificial neural network (ANN) is used. The ANN is a computational model 
which is based on biological neural network. It is used to extract the information from image with the help of interconnected group of artificial network. For training multi-layer artificial neural network Back Propagation Neural Network (BPNN) is commonly used. The fundamental arrangement of the BPNN presents 1 input layer and minimum 1 hidden layer followed by output layer. Figure 5 shows the model of Back Propagation Neural Network.

The detailed steps by using the BPNN ANN for Iris recognition are given below.

- Fill normalized Iris images data set which contains the feature vector values of different subjects and these are ranges from 0 to 1 .

- The normalized iris images obtained in previous section are used for training set and testing set by arbitrarily depiction out the data for training and testing.

- Generate 3 layers of iris normalized image an input, an output and a hidden layer. The dimension of the feature vector that characterizes the iris image information is equal to the number of nodes in the input.

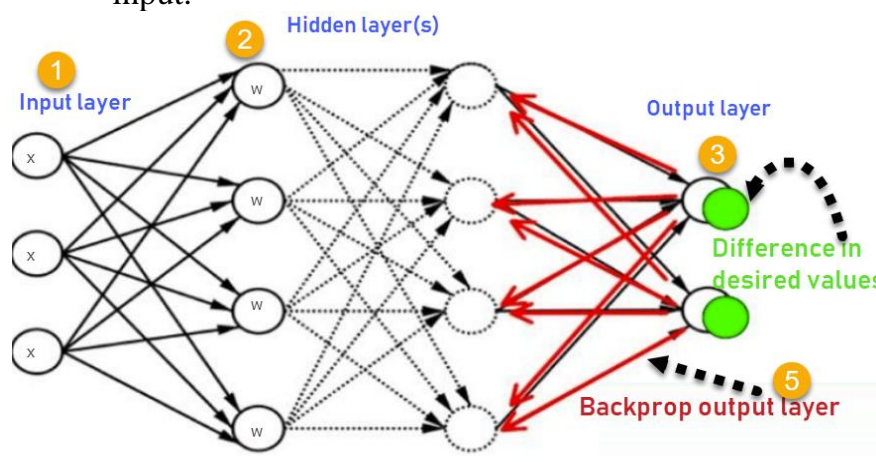

Fig.5 Model of Back Propagation Neural Network.

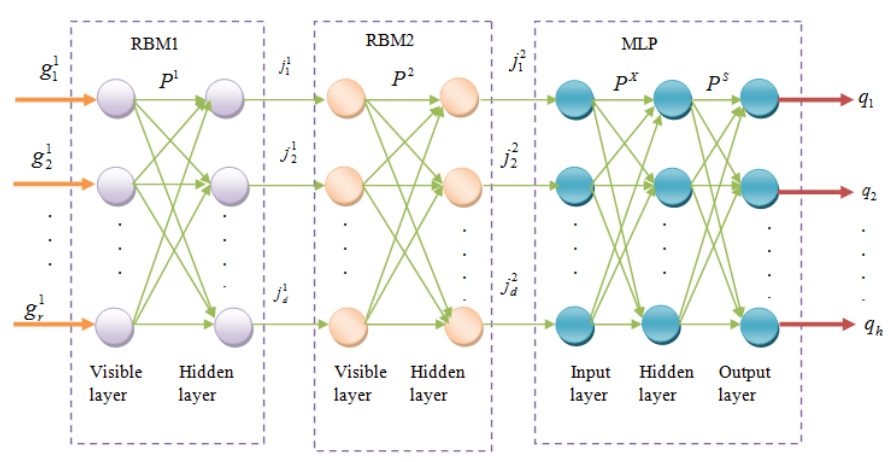

Fig.6 Architecture of DBN classifier

- Arbitrarily generates the nodes of the hidden layer. Arbitrarily generates all connection weights within a certain range. The output layer contains one node.

- Use Back Propagation algorithm to train the network. This algorithm is applied until the error is smallest amount for a certain number of training epochs specified by the user.

-Evaluate the performance and the test data to the trained network

\section{Chronological MBO-based DBN Neural Network}

The DBN classifier using the Chronological MBO algorithm is used for the identification of a person. The non-linear complex relation presents in the real life are removed by using DBN classifier and the chronological MBO algorithm trains the DBN classifier. The iris recognition is performed using the chronological MBO-based DBN, which is the integration of the chronological concept with the $\mathrm{MBO}$ algorithm to train DBN that depends on the migration features of the monarch butterfly. The standard MBO incorporates the fine tuning of parameters and the complex free computation to enhance the performance of the proposed chronological MBO-based DBN, and the high dimensional issues are effectively dealt using MBO. Figure 6 shows the Architecture of DBN classifier.

However, the searching speed and the convergence speed are enhanced by integrating the chronological concept, which defines the solutions (biases and weights) from the preceding iterations to revise the new biases and weights.

\section{Performance metrics}

The metrics used to evaluate the methods, are FRR, FAR, and accuracy are explained below.

1) Accuracy: The accuracy measures the accurateness of iris recognition based on iris modality and is represented as,

$$
\text { Accuracy }=\frac{J c+J u}{J c+R c+J u+R u}
$$

where, $J u$ denotes the true positives, and $J c$ is the true negatives. $R u$ denotes the false positives and $R c$ is the false negatives.

2) Sensitivity: Sensitivity is otherwise called True positive Rate (TPR), which is the measure of positive-ness identified correctly, and is calculated using the below equation.

Sensitivity $=\frac{J u}{R c+J u}$

3) False Rejection Ratio (FRR): FRR is the ratio of false rejection to the genuine attempts, and is expressed as,

$$
F R R=\frac{R c}{J u+R c}=1-T P R
$$

4) Specificity: Specificity or True Negative Rate (TNR) is the measure of false negatives, which are correctly located. Specificity is expressed as,

$$
\text { Specificity }=\frac{J c}{J c+R u}
$$

5) False Acceptance Rate (FAR): FAR is the ratio of false attempt to imposter attempts, and is represented as,

$$
F A R=\frac{R u}{R u+J c}=1-T N R
$$

6) Receiver Operating Characteristics (ROC): ROC refers to the relationship between TNR and TPR, which is used to compute the performance of the system. 


\section{BuILding Block Diagram OF PROJECTED STRUCTURE}

Here during this unit diagram of prompt structure is given. The implementation of this work is given in this step. Figure 7 shows suggest Method For Iris Recognition.

\section{Images Which are Test}

Here there are 20 people groups dataset is to be considered for the examination. The database utilized for this object is UBIRIS.V1 .V1 .V1.V4. The total database of this segment was searched for Iris pictures.

\section{Pre-processing and De-noising}

In pre-handling, the sign to-commotion portion of iris picture profile is upgraded additionally for this goal. Iris Recognition a ways off (IAAD) is utilized to improve the differentiation of the picture. Middle based channels are utilized to evacuate the sound introduces in the debased iris pictures.

\section{Hough Transform}

The Hough transform is used to extracts the different curves or shapes of an iris image.

\section{Segmentation and Normalization}

In this division step, the iris picture is part into disparate outskirts. The division of the districts can occur by considering comparable properties of an iris picture. A portion of the comparable properties are shading, brilliance, differentiate, surface, dark level, and so forth. The portioned iris picture is set up by utilizing a standardization calculation.

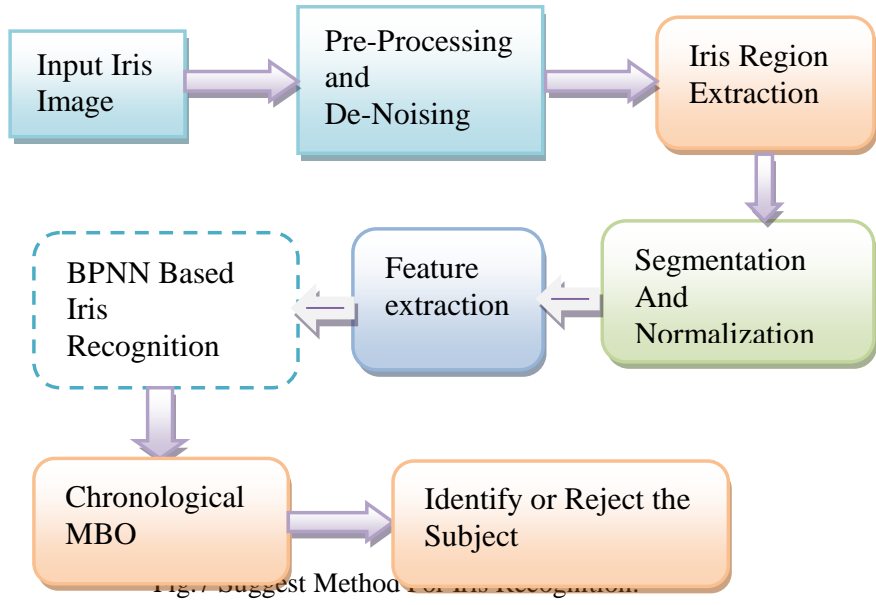

The fragmented iris picture is utilized for the future extraction process. Because of the shifting position of an individual and the camera, the iris picture is exceptionally influenced by mutilation [20]. Along these lines standardization is utilized to make up for this issue. The Daugman's Rubber Sheet Model is use for this reason.

\section{Feature Extraction}

For preparing multi-layer artificial neural system Back Propagation Neural Network (BPNN) is normally utilized. The major plan of the BPNN presents 1 info layer and least 1 shrouded layer pursued by yield layer.

The ScatT-Loop generator is use to remove the various highlights of an iris image.The ScatT-Loop creates the surface highlights for precise iris acknowledgment to remarkably recognize the people. Administrator in LGP uses the angle pixel esteems and is resolved as the force esteem. Distinctive changes shows in this progression are utilized to compute the surface highlights for the iris picture. PCA is an ideal plan to pack the high dimensional vectors into the low dimensional vectors and register the parameters from the information legitimately.

\section{Deep Belief Network (DBN)}

The dimensionally decreased highlights got utilizing PCA is bolstered as contribution to DBN to perceive the people. The powerful acknowledgment is acquired by the ideal tuning of the DBN classifier utilizing the Chronological MBO calculation. By utilizing Chronological MBO calculation we can gauge or characterize exact Iris picture.

\section{EXPERIMENTAL RESULTS}

Here observational results are characterized in this unit of proposed framework. The step by step execution of the proposed framework is displayed. Right off the bat we take info test iris picture. In this paper, there are Around 22,035 iris images from 700 individuals people group's dataset is to be considered for the trial. The database utilized for this design is UBIRIS.v1 [26]. Figure 3 demonstrates the key in iris acknowledgment picture having the measurement $512 \mathrm{x}$ 512. This picture can be chosen by utilizing the GUI in MatLab.

This info test picture of the iris is connected for the pre-processing. In this stage, the diverse commotion introduces in an iris picture are evacuated and increment the nature of a picture.

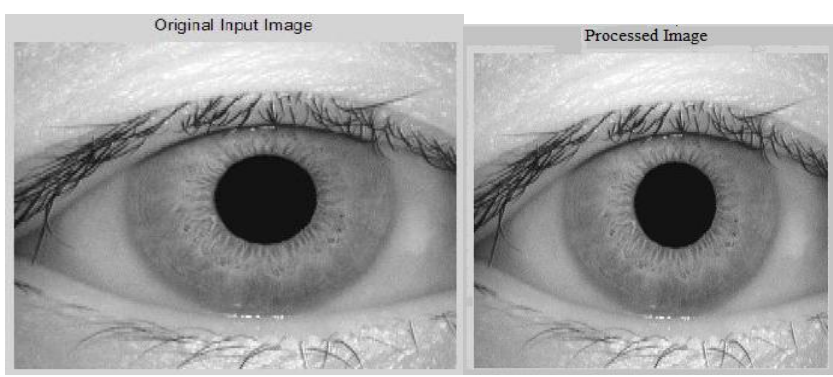

Fig.8 Input Iris Picture and Prossed Image.

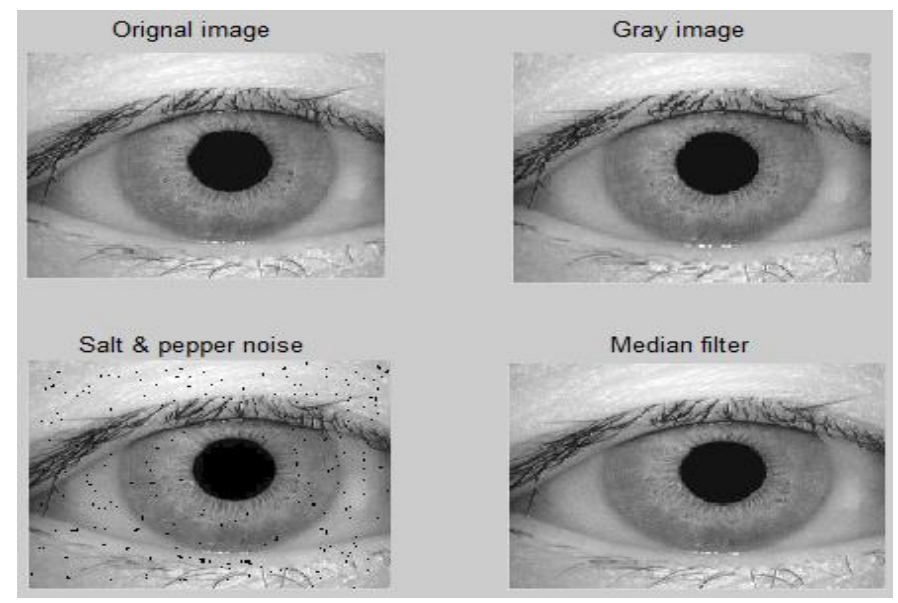

Published By: 
Fig.9 Operations On Iris Image

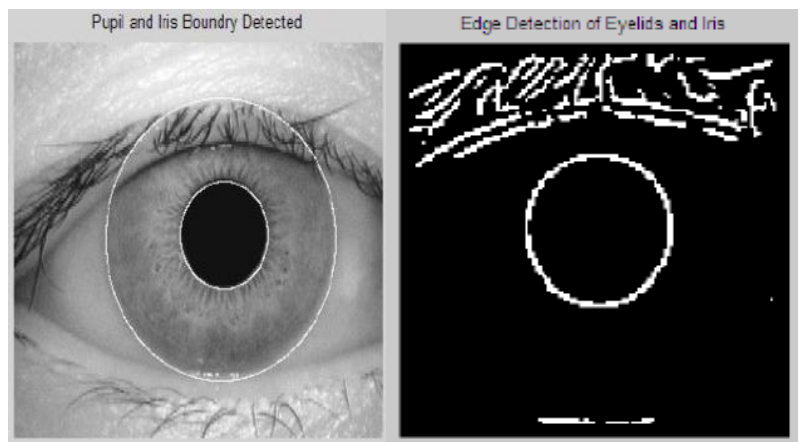

Fig. 10 Boundary detection of an Iris Image and Edge Detection Of An Iris Image.

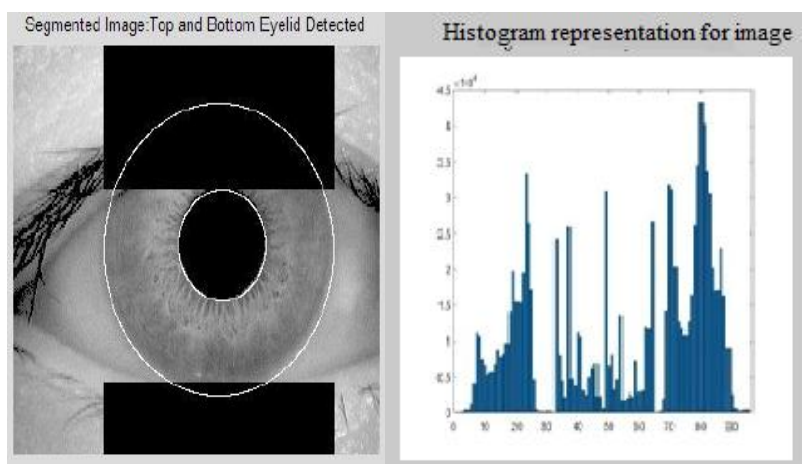

Fig.11 Eyelid of the bottom and top segmentation of an Iris Image and histogram representation for image.

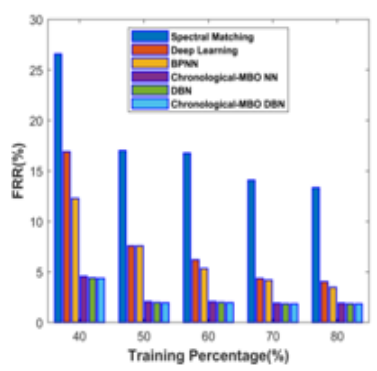

a)

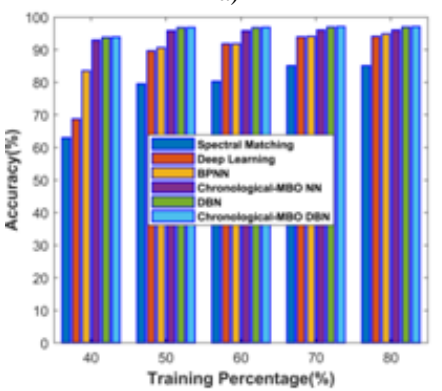

Fig. 12 Comparativbe analysis based on k-fold using image,a)FRR,b)FAR c)Accuracy and d) ROC analysis.

Figure 8 shows the input image and preprocessing image, which enhances the iris part of the eye (black portion). To eliminate the sound from iris images median filter is used. Figure 9 represents colors to gray as well as resounding image. Color image is the real image, besides of real image is gray image, salt also pepper sound is at the lower level of the original image, also the last one is the Median Filter image. In the Segmentation and Normalization process detection of Pupil and Iris boundary of the original image is takes place.
The detection is done with the help of Hough circles. Figure 10 shows the detected boundaries of an iris image. The white border presents in this image is the corresponding boundaries of the iris image. After boundary detection, the edge of an iris image is detected by using Normalization and Segmentation process.

Figure 11 shows the corresponding edges of the pupil of an iris image. For finding edge of canny edge detection algorithms are used to show the eyelid of the bottom and top segmentation of an Iris profile. Figure 12 depicts the comparative analysis for the metrics, a) shows FAR, b) shows accuracy, c) FRR and d) ROC analysis with respect to the $\mathrm{k}$-fold validation.

TABLE I. TABLE STYLES

\begin{tabular}{|l|l|l|l|l|l|l|}
\hline \multirow{2}{*}{ Methods } & \multicolumn{2}{|l|}{ FRR (\%) } & \multicolumn{2}{l|}{ FAR (\%) } & \multicolumn{2}{l|}{$\begin{array}{l}\text { Accuracy } \\
(\%)\end{array}$} \\
\cline { 2 - 7 } & $\begin{array}{l}\boldsymbol{k} \text {-fol } \\
\boldsymbol{d}\end{array}$ & Training & $\begin{array}{l}\boldsymbol{k} \text {-fol } \\
\boldsymbol{d}\end{array}$ & Training & $\begin{array}{l}\boldsymbol{k} \text {-fol } \\
\boldsymbol{d}\end{array}$ & Training \\
\hline $\begin{array}{l}\text { Spectral } \\
\text { Matching }\end{array}$ & 1.30 & 10.912 & 0.52 & 0.5964 & 85.0 & 63.002 \\
\hline $\begin{array}{l}\text { Deep } \\
\text { learning }\end{array}$ & 1.03 & 2.9 & 0.51 & 0.5261 & 86.3 & 68.764 \\
\hline BPNN & 0.85 & 0.786 & 0.51 & 0.5040 & 91.5 & 83.554 \\
\hline $\begin{array}{l}\text { Chronolo } \\
\text { gical-MB } \\
\text { O-NN }\end{array}$ & 0.5 & 0.786 & 0.5 & 0.5040 & 95.0 & 92.942 \\
\hline DBN & 0.47 & 0.745 & 0.49 & 0.4972 & 95.9 & 93.8 \\
\hline $\begin{array}{l}\text { Chronolo } \\
\text { gical-MB } \\
\text { O based } \\
\text { DBN }\end{array}$ & 0.47 & 0.4745 & 0.47 & 0.4889 & 96.0 & 93.886 \\
\hline
\end{tabular}

Table 1 demonstrates the comparative discussion of the existing and the proposed Chronological MBO-based DBN method. Based on $\mathrm{k}$-fold validation, the FRR rate obtained by the methods, spectral matching, deep learning, BPNN, Chronological-MBO-NN, DBN, and the proposed Chronological MBO based DBN is $1.3055 \%, 1.0372 \%$, $0.8577 \%, 0.5 \%, 0.4798 \%$, and $0.4745 \%$, respectively.

\section{CONCLUSION}

A powerful iris acknowledgment framework for individual distinguishing proof is available in this article. With the assistance of the double iris division false reaction is decreases. The pointless foundation pictures are additionally expelled with the assistance of picture division process. A technique for IAAD is presented for iris acknowledgment utilizing Chronological MBO-based DBN is proposed in this exploration work. The ideal loads are resolved to prepare the ordered MBO calculation utilizing DBN. Various strategies introduces in Hough change are utilized to for division, for example, straight, roundabout and illustrative. With the assistance of iris picture standardization the extraction of fixed number of highlights is finished. The UBIRIS.v1 database is utilized for testing reason for this iris acknowledgment framework. Additionally the Daugman model is utilized for iris division, standardization, include encoding and highlight coordinating. The iris acknowledgment dependent on the highlights of the biometric is performed utilizing the

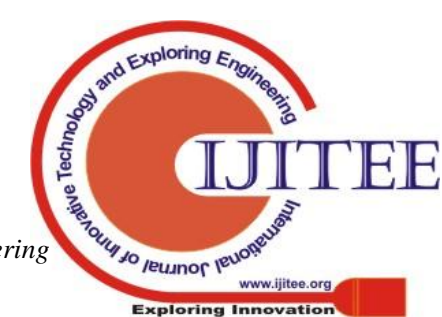


proposed Chronological MBO calculation, which is prepared utilizing the profound learning approach named as DBN. A robotized framework which is equipped for perceiving the iris is available in this paper. This framework decreases false reactions to brilliant sores and other retinal element of eye. The device utilized for this reason for existing is MatLab computerized picture handling. This paper presents iris acknowledgment framework that expels every past disadvantage. Likewise numerous issues of iris acknowledgment was illuminated like as human machine interface, picture handling and picture securing issues. The exhibition investigation demonstrate that the iris acknowledgment strategy is exact and effectively perceive the iris a good ways off 4 to 8 meter long. The proposed Chronological based DBN approach achieved insignificant FRR of $0.4745 \%$, negligible FAR of $0.4847 \%$, and maximal precision of $96.078 \%$. The future augmentation of this work can be made by utilizing any streamlining calculation to improve the presentation of iris acknowledgment.

\section{ACKNOWLEDGMENT}

The authors want to thank the Bharath Institute of Higher Education and Research, Chennai, Tamil Nadu, India. Also thanks to STE's NBN Sinhgad School of engineering, Ambegaon (Bk), Pune.

\section{REFERENCES}

[1] https://www.google.com/search?q=eye+crypts,+furrows, +stripes,+co ronas,+ freckles,+ etc $\&$ source $=\operatorname{lnm} \&$ tbm $=$ isch \&sa $=$ X\&ved $=0$ ahUKE wi_wefVsKfkAhWFWX0KHVDFAnUQ_AUIESgB\&biw=1366\&bi h=613\#imgdii=IvK7frn0Ri1nVM:\&imgrc=WLx8y3JatxPgPM:

[2] J. G. Daugman, "High confidence visual recognition of persons by a test of statistical independence", IEEE transactions on pattern analysis and machine intelligence, vol. 15, no. 11, 1993, pp. 1148-1161.

[3] L. Masek, "Recognition of human iris patterns for biometric identification", The University of Western Australia, vol. 2, no., 2003.

[4] W. W. Boles, and B. Boashash, "A human identification technique using images of the iris and wavelet transform", IEEE transactions on signal processing, vol. 46, no. 4, 1998, pp. 1185-1188.

[5] R. P. Wildes, J. C. Asmuth, G. L. Green et al., "A system for automated iris recognition", Applications of Computer Vision, 1994., Proceedings of the Second IEEE Workshop on, 1994, pp. 121-128.

[6] L. Ma, Y. Wang, and T. Tan, "Iris recognition using circular symmetric filters", Pattern Recognition, 2002. Proceedings. 16th International Conference on, 2002, pp. 414-417.

[7] A. E. Hassanien, A. Abraham, and C. Grosan, "Spiking neural network and wavelets for hiding iris data in digital images", Soft Computing, vol. 13 , no. 4, 2009, pp. 401-416.

[8] O. F. Soylemez, and B. Ergen, "Circular hough transform based eye state detection in human face images", Signal Processing and Communications Applications Conference (SIU), 2013 21st, 2013, pp. $1-4$.
[9] R. H. Abiyev, and K. Altunkaya, "Personal iris recognition using neural network", International Journal of Security and its Applications, vol. 2, no. 2, 2008, pp. 41- 50.

[10] L. F. Araghi, H. Shahhosseini, and F. Setoudeh, "IRIS recognition using neural network", Proceedings of the international multiconference of engineers and computer scientists, 2010.

[11] M. Gopikrishnan, and D. T. Santhanam, "A tradeoff between template size reduction and computational accuracy in Iris Patterns Recognition using Neural Networks", seec proceedings by, SEEC, vol., no., 2010.

[12] K. A. Raghavi, M. V. Priya, G. P. M. Paiva et al., "Human iris recognition using fuzzy neural concepts", The International Conference on Bioscience, Biochemistry and Bioinformatics (IPCBEE), 2011, pp. 256-260.

[13] C. Agurto, Y. Honggang, V. Murray, M.S. Pattichis, S. Barriga,W Bauman, et al., Detection of neo vascularization in the optic disc using an AM-FM representation, granulometry, and vessel segmentation, in: Annual International Conference of the IEEE, Engineering in Medicine and Biology Society (EMBC), 2012, pp. 4946-4949.

[14] G. Fahmy, "Super-resolution construction of iris image low resolution face video," in Signal Processing and Its Applications, 2007. ISSPA 2007. 9th International Symposium.

[15] Yap, P. T., Raveendran, P., \& Ong, S. H. (2003). Image analysis by Krawtchouk moments. IEEE Transactions on Image Processing, 12(11), 1367-1377.

\section{AUTHORS PROFILE}

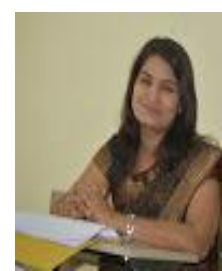

: Ms.Swati D. Shirke holds an M.E.degree in Computer Science and Engineering from Pune University and is a research fellow in the Department of Computer Science and Engineering, Bharath Institute of Higher Education and Research, Bharath University. Her main area of interest includes pattern recognition, image processing, machine learning, She has published several papers in well known peer-reviewed journals.

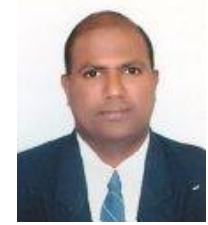

Dr. C. Rajabhushnam holds a Ph.D. in Neura Networks, from Louisiana State University, USA And he is a professor in the Department of In the Department of Computer Science and Engineering, Bharath Institute of Higher Education and Research, Bharath University. His main area of interest includes pattern recognition, image processing, and machine learning he has published several papers in well known peer-reviewed journals. 